\title{
Deformations of Period Lattices of Flexible Polyhedral Surfaces
}

\author{
Alexander A. Gaifullin • Sergey A. Gaifullin
}

Received: 2 June 2013 / Revised: 13 January 2014 / Accepted: 18 January 2014 /

Published online: 22 February 2014

(C) Springer Science+Business Media New York 2014

\begin{abstract}
At the end of the 19th century Bricard discovered the phenomenon of flexible polyhedra, that is, polyhedra with rigid faces and hinges at edges that admit nontrivial flexes. One of the most important results in this field is a theorem of Sabitov, asserting that the volume of a flexible polyhedron is constant during the flexion. In this paper we study flexible polyhedral surfaces in $\mathbb{R}^{3}$, doubly periodic with respect to translations by two non-collinear vectors, that can vary continuously during the flexion. The main result is that the period lattice of a flexible doubly periodic surface that is homeomorphic to the plane cannot have two degrees of freedom.
\end{abstract}

Keywords Flexible polyhedra $\cdot$ Polyhedral surfaces $\cdot$ Places of fields

\author{
A.A. Gaifullin \\ Steklov Mathematical Institute, Moscow, Russia \\ e-mail: agaif@mi.ras.ru
}

\author{
A.A. Gaifullin · S.A. Gaifullin \\ Lomonosov Moscow State University, Moscow, Russia
}

S.A. Gaifullin

e-mail: sgayf@yandex.ru

\section{A.A. Gaifullin}

Kharkevich Institute for Information Transmission Problems, Moscow, Russia

\author{
A.A. Gaifullin \\ Demidov Yaroslavl State University, Yaroslavl, Russia
}

S.A. Gaifullin

Higher School of Economics, Moscow, Russia 
Fig. 1 Simplest examples of flexible polyhedral surfaces
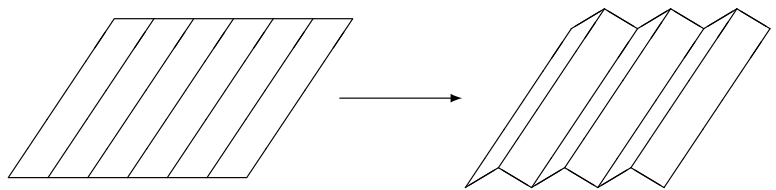

$a$
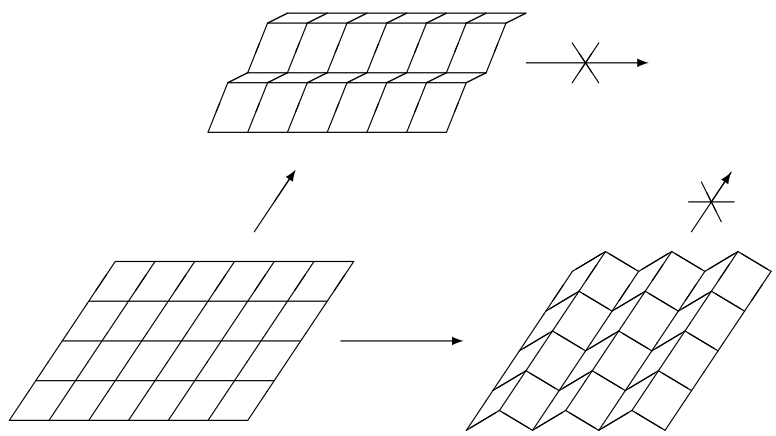

$b$

\section{Introduction}

We denote by $\mathbb{R}^{3}$ the three-dimensional Euclidean space. Let $S \subset \mathbb{R}^{3}$ be a polyhedral surface that is homeomorphic to a plane and is situated "near" the horizontal plane $\mathbb{R}^{2}$. Suppose that $S$ has rigid faces and hinges at the edges. This means that $S$ is allowed to flex so that the faces remain congruent to themselves, while the dihedral angles at the edges change continuously. For example, let $S$ be the horizontal plane, $z=0$, with hinges at the lines $y=k, k \in \mathbb{Z}$. Then $S$ can be shrunk in the direction of the $x$-axis, as is shown in Fig. 1(a). Now, let $S$ be the horizontal plane with hinges at both the lines $y=k$ and the lines $x=k, k \in \mathbb{Z}$. Then $S$ can be shrunk either in the direction of the $x$-axis, or in the direction of the $y$-axis. However, once we have already started to shrink $S$ in the direction of the $x$-axis, we are not able anymore to shrink it in the direction of the $y$-axis, and vice versa; see Fig. 1(b). In this paper we consider the following natural question.

Is it possible to construct a polyhedral surface $S \subset \mathbb{R}^{3}$ that can be shrunk near the horizontal plane independently in two different directions? In other words: is it possible that there exists a two-parametric flexion of $S$ such that, on varying the first parameter, we shrink $S$ in the first direction, and on varying the second parameter, we shrink $S$ in the second direction?

The authors are indebted to the architect Sergei Kolchin who suggested this question to them.

In this paper we focus on the case of a doubly periodic polyhedral surface, i.e., a polyhedral surface $S$ invariant under the translations by two non-collinear vectors $a$ and $b$. We allow the surface $S$ to flex so that it remains doubly periodic and the period vectors $a$ and $b$ change continuously. During the last years, flexible periodic frameworks have been studied in $[1,6,8,12]$. 
Now we proceed with some rigorous definitions and results.

Definition 1.1 An embedded polyhedral surface is a surface $S \subset \mathbb{R}^{3}$ without boundary, consisting of polygons glued along their sides. These polygons are called faces of $S$.

Definition 1.2 An embedded doubly periodic polyhedral surface is a triple $(S, a, b)$ such that $S \subset \mathbb{R}^{3}$ is an embedded polyhedral surface, and $a, b \in \mathbb{R}^{3}$ are non-collinear vectors such that

(1) $S$ is invariant under the translations by $a$ and $b$,

(2) the action of the lattice $\Lambda$ generated by $a$ and $b$ on the surface $S$ is cocompact, i.e., the quotient $S / \Lambda$ is compact.

In this definition we do not require that $S$ be homeomorphic to the plane. If $S$ is homeomorphic to the plane, then condition (2) follows from condition (1). Indeed, $S / \Lambda$ must be homeomorphic to the torus, since it is a two-dimensional manifold without boundary with fundamental group $\mathbb{Z} \oplus \mathbb{Z}$.

Until Sect. 3 we consider only embedded polyhedral surfaces, and we omit the word "embedded".

Definition 1.3 A flex of a doubly periodic polyhedral surface $(S, a, b)$ is a continuous deformation $(S(t), a(t), b(t)), t \in[0,1], S(0)=S, a(0)=a, b(0)=b$, such that, for each $t$, the triple $(S(t), a(t), b(t))$ is a doubly periodic polyhedral surface and every face of $S(t)$ is congruent to the corresponding face of $S$.

Up to rotations of $\mathbb{R}^{3}$ the period lattice $\Lambda$ of the doubly periodic polyhedral surface $(S, a, b)$ is determined by the Gram matrix $G$ of the vectors $a$ and $b$. We are interested in the deformations of the period lattice $\Lambda$ under flexes. However, we would like to neglect rotations. So we are interested in the deformations of the Gram matrix $G$ induced by flexions of $(S, a, b)$. The main result of the present paper says that if $S$ is homeomorphic to a plane, then only one-parametric deformations of $G$ may occur. This means that we cannot find a two-parametric flexion that yields a truly two-parametric deformation of $G$. This can be formulated more formally as follows. Consider all possible doubly periodic polyhedral surfaces $\left(S^{\prime}, a^{\prime}, b^{\prime}\right)$ that can be obtained from $(S, a, b)$ by flexions. Let $\mathcal{G}=\mathcal{G}(S, a, b)$ be the set of all matrices $G^{\prime}$ that appear as the Gram matrices of the vectors $a^{\prime}, b^{\prime}$ for such doubly periodic surfaces $\left(S^{\prime}, a^{\prime}, b^{\prime}\right)$.

Theorem 1.4 Let $(S, a, b)$ be an embedded doubly periodic polyhedral surface homeomorphic to a plane. Then the set $\mathcal{G}(S, a, b)$ is contained in a one-dimensional real affine algebraic variety.

This theorem can be generalized to the case of a non-embedded polyhedral surface; see Theorem 4.1 .

Interest in results of this kind originated with the famous result of Sabitov $[9,10]$ that the volume of a (compact) flexible polyhedron in $\mathbb{R}^{3}$ remains constant under the 
Fig. 2 Doubly periodic connected sum of the plane with flexible polyhedra

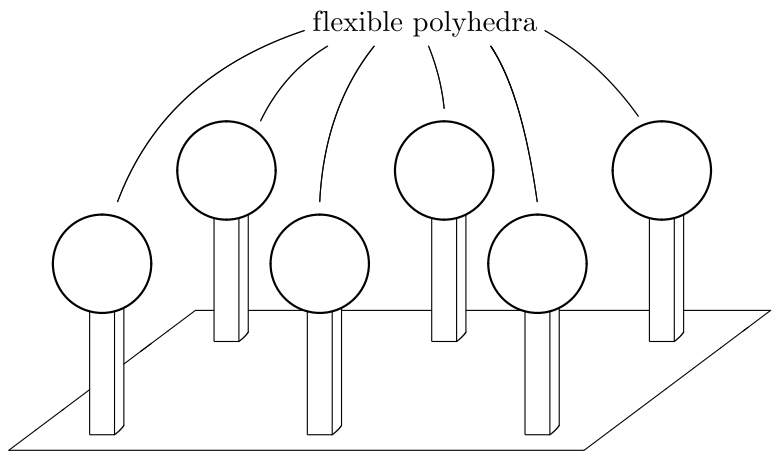

flexion. Sabitov obtained this result by proving that the volume $V$ of every simplicial polyhedron satisfies a polynomial relation of the form

$$
V^{2 N}+a_{1}(\ell) V^{2 N-2}+\cdots+a_{N}(\ell)=0
$$

where $a_{i}(\ell)$ are polynomials with rational coefficients in the squares of the edge lengths of the polyhedron. (The number $N$ and the polynomials $a_{i}$ depend on the combinatorial structure of the polyhedron.) The same result for polyhedra of dimensions $n \geq 4$ has recently been obtained by one of the authors [3, 4].

A natural question is which other invariants of polyhedra satisfy polynomial relations of the form (1). For a doubly periodic polyhedral surface, the natural invariants are the coefficients $g_{11}, g_{12}$, and $g_{22}$ of the Gram matrix of the period lattice. Though we cannot obtain a polynomial relation of the form (1) for any of these coefficients, we shall prove that the three coefficients $g_{11}, g_{12}$, and $g_{22}$, and the set $\ell$ of the squares of edge lengths are subject to two polynomial relations such that, for any given $\ell$, these relations yield an affine variety of dimension not greater than 1 .

Some ideas of our proof of Theorem 1.4 are inspired by Sabitov's theorem, and especially by another proof of Sabitov's theorem obtained by Connelly, Sabitov, and Walz [2]. The main tool is the theory of places (see Sect. 3). It is standard to use places to prove that a certain element is integral over the given ring. Our situation is more difficult, namely, we need to prove that among the three given elements $g_{11}, g_{12}$, and $g_{22}$ there exist at least two independent relations over the given ring.

Notice that we can easily construct doubly periodic polyhedral surfaces with an arbitrarily large number of degrees of freedom by taking connected sums of the plane with flexible polyhedra (see Fig. 2). Such flexes can be called 'local', since they do not change the surface off some small disks, and, in particular, do not affect the period lattice. Theorem 1.4 describes the 'global behavior' of flexible doubly periodic polyhedral surfaces.

Now, we would like to formulate two natural open questions.

Question 1.5 Does the assertion of Theorem 1.4 hold for an arbitrary doubly periodic polyhedral surface, not necessarily homeomorphic to a plane? 
Question 1.6 Let $\left(S, a_{1}, \ldots, a_{k}\right)$ be a $k$-dimensional $k$-periodic polyhedral submanifold in $\mathbb{R}^{n}$. What is the maximal possible dimension of $\mathcal{G}\left(S, a_{1}, \ldots, a_{k}\right)$ ?

\section{Examples}

The set $\mathcal{G}(S, a, b)$ depends not only on the polyhedral surface $S$, but also on the vectors $a$ and $b$. If $\tilde{a}, \tilde{b}$ is another basis of the same lattice $\Lambda,(\tilde{a}, \tilde{b})=(a, b) C$, then the usual formula $\widetilde{G}=C^{T} G C$ provides a canonical affine isomorphism of the sets $\mathcal{G}(S, a, b)$ and $\mathcal{G}(S, \tilde{a}, \tilde{b})$. We shall identify them and denote this set by $\mathcal{G}(S, \Lambda)$.

Now, let us consider a sublattice $\tilde{\Lambda}$ of the lattice $\Lambda$. Let $a, b$ be a basis of $\Lambda$ and let $\tilde{a}, \tilde{b}$ be a basis of $\tilde{\Lambda}$. Then the same formula, $\widetilde{G}=C^{T} G C$, yields the affine embedding $\mathcal{G}(S, \Lambda) \hookrightarrow \mathcal{G}(S, \widetilde{\Lambda})$. Nevertheless, this embedding is not necessarily an isomorphism, since the polyhedral surface $S$ may have flexes $S(t)$ such that $S(t)$ remains doubly periodic with respect to a continuous deformation $\widetilde{\Lambda}(t)$ of the lattice $\widetilde{\Lambda}$, but it does not remain doubly periodic with respect to any continuous deformation of the lattice $\Lambda$.

Example 2.1 Consider a plane and fix a point $p$ in it and two non-collinear vectors $a$ and $b$ parallel to it. Let us divide this plane into triangles by the straight lines parallel to $a$ through the points $p+k b, k \in \mathbb{Z}$, the straight lines parallel to $b$ through the points $p+k a, k \in \mathbb{Z}$, and the straight lines parallel to $a-b$ through the points $p+k a, k \in \mathbb{Z}$. Let $S$ be the polyhedral surface consisting of all these triangles. It is easy to see that the doubly periodic polyhedral surface $(S, \Lambda)$ is not flexible, where $\Lambda=\mathbb{Z} a+\mathbb{Z} b$.

However, the surface $S$ will become flexible if we replace $\Lambda$ by a sublattice of it.

Example 2.2 Consider the same surface $S$ and the period lattice $\tilde{\Lambda}=\mathbb{Z} a+2 \mathbb{Z} b$. Then the doubly periodic polyhedral surface $(S, \widetilde{\Lambda})$ admits only one type of flexion. We can shrink it in the direction orthogonal to $a$. Then for the Gram matrix of the basis of $\tilde{\Lambda}$, we have $g_{11}=$ const, $g_{12}=$ const , and $g_{22}$ varies from $\frac{4\langle a, b\rangle^{2}}{|a|^{2}}$ to $4|b|^{2}$. Hence $\mathcal{G}(S, \widetilde{\Lambda})$ is a segment.

Example 2.3 Consider the same surface $S$ and the period lattice $\widehat{\Lambda}=2 \mathbb{Z} a+2 \mathbb{Z} b$. Then we can shrink $(S, \widehat{\Lambda})$ in the direction orthogonal to $a$, in the direction orthogonal to $b$, or in the direction orthogonal to $a-b$. Hence $\mathcal{G}(S, \widehat{\Lambda})$ contains three segments. (We do not claim that $\mathcal{G}(S, \widehat{\Lambda})$ consists only of these three segments.)

Example 2.4 Now, let us give an example of $(S, \Lambda)$ such that the Zariski closure of $\mathcal{G}(S, \Lambda)$ has an irreducible component which is not a straight line. This example is a well-known flexible surface consisting of parallelograms (see Fig. 3). Initially the surface is flat. Then it can be folded so that the full lines are mountain foldings and the dashed lines are valley foldings, cf. [11]. Such a folding was known in the ancient Japanese origami technique and is called the Miura-ori folding after Miura, who suggested applying this type of folding to solar panels. The polyhedral surface $S$ shown in Fig. 3 will be considered with the period lattice $\Lambda=\mathbb{Z} a+\mathbb{Z} b$. 
Fig. 3 The Miura-ori folding

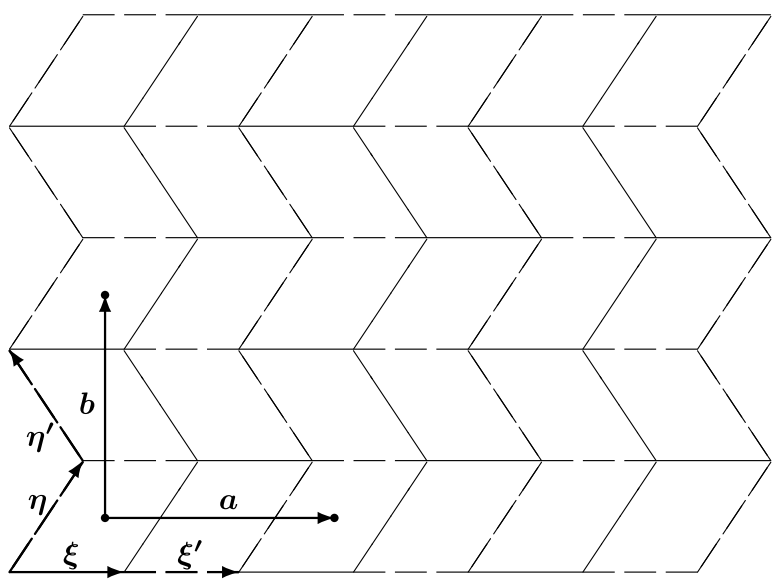

Since the period lattice $\Lambda$ changes during the flexion, we shall further denote the initial basis vectors $a$ and $b$ by $a_{0}$ and $b_{0}$, respectively, and we shall use the notation $a$ and $b$ for the period vectors after the flex. Let $\alpha$ be the acute angle of a parallelogram of $S$. Take the orthonormal basis $e_{1}=\frac{a_{0}}{\left|a_{0}\right|}, e_{2}=\frac{b_{0}}{\left|b_{0}\right|}, e_{3}$ in $\mathbb{R}^{3}$. Since $S$ is doubly periodic, there are four classes of equal vectors of edges of $S$ with representatives $\xi$, $\eta$, $\xi^{\prime}$, and $\eta^{\prime}$; see Fig. 3. The initial positions of the vectors $\xi$ and $\eta$ are the vectors $\xi_{0}=\left(\frac{\left|a_{0}\right|}{2}, 0,0\right)$ and $\eta_{0}=\left(\frac{1}{2}\left|b_{0}\right| \cot \alpha, \frac{\left|b_{0}\right|}{2}, 0\right)$.

We shall show that $S$ admits a flexion such that the period vectors $a$ and $b$ remain parallel to $a_{0}$ and $b_{0}$, respectively. We shall find a relation between $|a|$ and $|b|$ that is satisfied under this flexion. Let us give the flexion by

$$
\begin{array}{ll}
\xi=\left(\frac{|a|}{2}, 0, z\right), & \xi^{\prime}=\left(\frac{|a|}{2}, 0,-z\right), \\
\eta=\left(x, \frac{|b|}{2}, 0\right), & \eta^{\prime}=\left(-x, \frac{|b|}{2}, 0\right),
\end{array}
$$

where $x=\frac{\left|a_{0}\right|\left|b_{0}\right| \cot \alpha}{2|a|}, z=\frac{\sqrt{\left|a_{0}\right|^{2}-|a|^{2}}}{2}$. It is easy to see that $a$ and $b$ are in fact period vectors of $S$. Let us write down the conditions that the parallelograms of $S$ remain congruent to themselves during the flexion. It is easy to see that these conditions for all parallelograms follow from the conditions for the parallelogram with sides $\xi$ and $\eta$. We have

$$
\begin{aligned}
& \langle\xi, \xi\rangle=\frac{|a|^{2}}{4}+z^{2}=\frac{\left|a_{0}\right|^{2}}{4}=\left\langle\xi_{0}, \xi_{0}\right\rangle, \\
& \langle\xi, \eta\rangle=\frac{1}{2}|a| x=\frac{1}{4}\left|a_{0}\right|\left|b_{0}\right| \cot \alpha=\left\langle\xi_{0}, \eta_{0}\right\rangle .
\end{aligned}
$$

The condition $\langle\eta, \eta\rangle=\left\langle\eta_{0}, \eta_{0}\right\rangle$ gives

$$
x^{2}+\frac{|b|^{2}}{4}=\frac{\left|b_{0}\right|^{2}}{4 \sin ^{2} \alpha} .
$$


Hence,

$$
|a|^{2}|b|^{2} \sin ^{2} \alpha-|a|^{2}\left|b_{0}\right|^{2}+\left|a_{0}\right|^{2}\left|b_{0}\right|^{2} \cos ^{2} \alpha=0 .
$$

Therefore, the Gram matrix elements of the period vectors $a$ and $b$ satisfy the system of equations

$$
\left\{\begin{array}{l}
g_{11} g_{22} \sin ^{2} \alpha-g_{11}\left|b_{0}\right|^{2}+\left|a_{0}\right|^{2}\left|b_{0}\right|^{2} \cos ^{2} \alpha=0, \\
g_{12}=0
\end{array}\right.
$$

Thus, $\mathcal{G}(S, \Lambda)$ contains a segment of a hyperbola.

Certainly, $\mathcal{G}(S, \Lambda)$ also contains a straight segment, corresponding to the shrinking in the direction orthogonal to $a$.

\section{Notation}

We recall some basic facts on places of fields. For more detailed information and proofs, see [5, 7].

Suppose $L$ and $F$ are fields. Let us add an extra element $\infty$ to the field $F$. The elements of $F$ are called finite elements of $F \cup\{\infty\}$. Extend the addition and the multiplication in $F$ to partially defined operations in $F \cup\{\infty\}$ by

$$
\begin{array}{ll}
z+\infty=\infty+z=\infty & \text { for all } z \in F \\
z \cdot \infty=\infty \cdot z=\infty & \text { for all } z \in(F \backslash\{0\}) \cup\{\infty\}
\end{array}
$$

The expressions $\infty+\infty, \infty \cdot 0$, and $0 \cdot \infty$ are undefined.

Definition 3.1 A mapping $\varphi: L \rightarrow F \cup\{\infty\}$ is called a place of $L$ into $F$ if for all $x, y \in L$ we have

(1) $\varphi(x+y)=\varphi(x)+\varphi(y)$,

(2) $\varphi(x y)=\varphi(x) \varphi(y)$,

(3) $\varphi(1)=1$

whenever the right-hand sides are defined.

It follows immediately from the definition that $\varphi(0)=0$ for any place $\varphi$. The following properties of places are also standard.

Lemma 3.2 Let $\varphi: L \rightarrow F \cup\{\infty\}$ be a place and let $x \in$ L. Then

(1) $\varphi(x)=\infty$ if and only if $\varphi(-x)=\infty$,

(2) $\varphi(x)=\infty$ if and only if $\varphi\left(x^{-1}\right)=0$, where $x \neq 0$.

Proof (1) Assume that $\varphi(x)=\infty$ and $\varphi(-x) \neq \infty$. Then $\varphi(x)+\varphi(-x)$ is defined and equals $\infty$. Hence $\varphi(0)=\infty$, which yields a contradiction.

(2) Assume that $\varphi(x)=\infty$ and $\varphi\left(x^{-1}\right) \neq 0$. Then $\varphi(x) \cdot \varphi\left(x^{-1}\right)$ is defined and equals $\infty$. Hence $\varphi(1)=\infty$, which yields a contradiction. The opposite implication is similar. 
It is clear that the restriction of a place to a subfield is a place, and the composition of places is a place. (Taking the composition of places $\varphi: L \rightarrow F \cup\{\infty\}$ and $\psi: F \rightarrow$ $E \cup\{\infty\}$, we always put $\psi(\infty)=\infty$.)

The following basic fact on places can be found in [5, Chap. I, Theorem 1].

Lemma 3.3 Let $L$ be a field containing a ring $R$. Let $\psi$ be a homomorphism of $R$ into a field $F$, and suppose $\psi(1)=1$. Then $\psi$ can be extended to a place $L \rightarrow \bar{F} \cup\{\infty\}$, where $\bar{F}$ is the algebraic closure of $F$.

Definition 3.4 A mapping of a simplicial complex $K_{1}$ to a simplicial complex $K_{2}$ is called simplicial if it maps every simplex of $K_{1}$ linearly onto a simplex of $K_{2}$. An action of a discrete group $\Lambda$ on a simplicial complex $K$ is called simplicial if every element of $\Lambda$ acts by a simplicial mapping $K \rightarrow K$.

Let $K$ be a simplicial complex homeomorphic to a plane with a free simplicial action of the group $\Lambda \cong \mathbb{Z} \oplus \mathbb{Z}$ with generators $\alpha$ and $\beta$. We shall use the additive notation for the group $\Lambda$. For an element $\lambda \in \Lambda$, we denote by $T_{\lambda}$ the corresponding simplicial automorphism of $K$. Then we have $T_{\lambda} T_{\mu}=T_{\lambda+\mu}$.

Definition 3.5 Consider a mapping $\theta: K \rightarrow \mathbb{R}^{3}$, linear on simplices of $K$, and equivariant with respect to an action of $\Lambda$ on $\mathbb{R}^{3}$ such that $\alpha$ and $\beta$ act by translations by some vectors $a=a(\theta)$ and $b=b(\theta)$. Then the pair $(K, \theta)$ is called a doubly periodic polyhedral surface. If $\theta$ is injective, the polyhedral surface $(K, \theta)$ is called embedded.

This definition of an embedded doubly periodic polyhedral surface is equivalent to Definition 1.2.

Let $v_{1}, \ldots, v_{n}$ be representatives of all $\Lambda$-orbits of the vertices of $K$. Consider the field

$$
\mathbb{L}=\mathbb{L}(K, \Lambda)=\mathbb{Q}\left(x_{\alpha}, y_{\alpha}, z_{\alpha}, x_{\beta}, y_{\beta}, z_{\beta}, x_{v_{1}}, y_{v_{1}}, z_{v_{1}}, \ldots, x_{v_{n}}, y_{v_{n}}, z_{v_{n}}\right),
$$

where $x_{\alpha}, y_{\alpha}, z_{\alpha}, x_{\beta}, y_{\beta}, z_{\beta}, x_{v_{1}}, y_{v_{1}}, z_{v_{1}}, \ldots, x_{v_{n}}, y_{v_{n}}, z_{v_{n}}$ are independent variables over $\mathbb{Q}$. For $\lambda=m \alpha+k \beta$, we set $x_{\lambda}=m x_{\alpha}+k x_{\beta}, y_{\lambda}=m y_{\alpha}+k y_{\beta}, z_{\lambda}=m z_{\alpha}+k z_{\beta}$. For $u=T_{\lambda}\left(v_{i}\right)$, we set $x_{u}=x_{\lambda}+x_{v_{i}}, y_{u}=y_{\lambda}+y_{v_{i}}, z_{u}=z_{\lambda}+z_{v_{i}}$. It is not hard to show that the field $\mathbb{L}(K, \Lambda)$ is independent of the choice of the representatives $v_{1}, \ldots, v_{n}$ and the generators $\alpha$ and $\beta$ of $\Lambda$.

If we have a doubly periodic polyhedral surface $(K, \theta)$, then we obtain the specialization homomorphism

$$
\tau_{\theta}: \mathbb{Q}\left[x_{\alpha}, y_{\alpha}, z_{\alpha}, x_{\beta}, y_{\beta}, z_{\beta}, x_{v_{1}}, y_{v_{1}}, z_{v_{1}}, \ldots, x_{v_{n}}, y_{v_{n}}, z_{v_{n}}\right] \rightarrow \mathbb{R}
$$

that takes $x_{u}, y_{u}$, and $z_{u}$ to the coordinates of the point $\theta(u)$ for every vertex $u$, that takes $x_{\alpha}, y_{\alpha}$, and $z_{\alpha}$ to the coordinates of the vector $a$, and that takes $x_{\beta}, y_{\beta}$, and $z_{\beta}$ to the coordinates of the vector $b$.

We shall find it convenient to identify a vertex $v$ of $K$ with a point $\left(x_{v}, y_{v}, z_{v}\right) \in \mathbb{L}^{3}$. We shall also identify an element $\lambda \in \Lambda$ with a vector $\left(x_{\lambda}, y_{\lambda}, z_{\lambda}\right) \in \mathbb{L}^{3}$. Then $T_{\lambda}(v)$ is identified with $v+\lambda$. Thus we obtain the linear embedding $\Lambda \subset \mathbb{L}^{3}$. 
We endow the vector space $\mathbb{L}^{3}$ with the standard inner product given by

$$
\langle\xi, \eta\rangle=\xi_{1} \eta_{1}+\xi_{2} \eta_{2}+\xi_{3} \eta_{3}, \quad \xi=\left(\xi_{1}, \xi_{2}, \xi_{3}\right), \eta=\left(\eta_{1}, \eta_{2}, \eta_{3}\right)
$$

We introduce the following elements of the field $\mathbb{L}$ :

$$
\begin{aligned}
& g_{11}=\langle\alpha, \alpha\rangle, \quad g_{12}=\langle\alpha, \beta\rangle, \quad g_{22}=\langle\beta, \beta\rangle, \\
& \ell_{u v}=\langle v-u, v-u\rangle,
\end{aligned}
$$

where $u$ and $v$ are vertices of $K$.

For any doubly periodic polyhedral surface $(K, \theta)$, the corresponding homomorphism $\tau_{\theta}$ takes $g_{11}, g_{12}$, and $g_{22}$ to the elements of the Gram matrix of the vectors $a(\theta)$ and $b(\theta)$, and it takes $\ell_{u v}$ to the square of the distance between $\theta(u)$ and $\theta(v)$.

Let $R=R(K, \Lambda)$ be a $\mathbb{Q}$-subalgebra of the field $\mathbb{L}$ generated by all $\ell_{u v}$ such that $[u v]$ is an edge of $K$.

\section{Main Result}

Fix $K$ and a set of numbers $l=\left\{l_{u v}\right\}, l_{u v}=l_{v u} \in \mathbb{R}$, where [uv] runs over all edges of $K$. Consider all possible doubly periodic polyhedral surfaces $(K, \theta)$ with the set of the squares of edge lengths equal to $l$. Let $\mathcal{G}=\mathcal{G}(K, \Lambda, l)$ be the set of all matrices $G$ that appear as the Gram matrices of the vectors $a(\theta), b(\theta)$ for such doubly periodic surfaces $(K, \theta)$. (If $l$ cannot be realized as the set of the squares of the edge lengths of a polyhedral surface, then the set $\mathcal{G}(K, \Lambda, l)$ is empty.) The set $\mathcal{G}$ is contained in the three-dimensional affine space $\mathbb{R}^{3}$ with coordinates $g_{11}, g_{12}, g_{22}$.

The following theorem is a strengthened version of Theorem 1.4 for polyhedral surfaces that are not necessarily embedded.

Theorem 4.1 Let $K$ be a simplicial complex homeomorphic to $\mathbb{R}^{2}$ with a free simplicial action of the group $\Lambda \cong \mathbb{Z} \oplus \mathbb{Z}$ with generators $\alpha$ and $\beta$. Then for each set of numbers $l=\left\{l_{u v}\right\}, l_{u v}=l_{v u} \in \mathbb{R}$, there is a one-dimensional real affine algebraic subvariety of $\mathbb{R}^{3}$ containing $\mathcal{G}(K, \Lambda, l)$.

To prove this theorem we shall study polynomial relations among the elements $g_{11}, g_{12}, g_{22} \in \mathbb{L}=\mathbb{L}(K, \Lambda)$ with coefficients in the ring $R=R(K, \Lambda)$. Any such polynomial relation has the form $f\left(g_{11}, g_{12}, g_{22}\right)=0$, where $f \in R[X, Y, Z]$. The free $R$-algebra $R[X, Y, Z]$ has a natural $\mathbb{Z}$-grading given by $\operatorname{deg} X=\operatorname{deg} Y=$ $\operatorname{deg} Z=1$. For each $f \in R[X, Y, Z], f \not \equiv 0$, we denote by $\widehat{f}$ the homogeneous component of $f$ of maximal degree.

Proposition 4.2 Let $K$ be a simplicial complex homeomorphic to $\mathbb{R}^{2}$ with a free simplicial action of a group $\Lambda \cong \mathbb{Z} \oplus \mathbb{Z}$ with generators $\alpha$ and $\beta, \mathbb{L}=\mathbb{L}(K, \Lambda)$, and $R=R(K, \Lambda)$. Then the elements $g_{11}, g_{12}, g_{22} \in \mathbb{L}$ satisfy a system of two non-trivial polynomial equations,

$$
\left\{\begin{array}{l}
f\left(g_{11}, g_{12}, g_{22}\right)=0 \\
h\left(g_{11}, g_{12}, g_{22}\right)=0
\end{array}\right.
$$


with coefficients in $R$ such that $\widehat{f}$ and $\widehat{h}$ have coefficients in $\mathbb{Q}$, and $\widehat{f}$ and $\widehat{h}$ are coprime in $\mathbb{Q}[X, Y, Z]$.

Proof Let us formulate a key lemma.

Lemma 4.3 Let $K$ be a simplicial complex homeomorphic to $\mathbb{R}^{2}$ with a free simplicial action of a group $\Lambda \cong \mathbb{Z} \oplus \mathbb{Z}$, and let $\mathbb{L}=\mathbb{L}(K, \Lambda)$. Let $\varphi: \mathbb{L} \rightarrow F \cup\{\infty\}$ be a place such that char $F \neq 2$ and $\varphi\left(\ell_{u v}\right) \neq \infty$ for all edges $[u v]$ of $K$. Then there exists a basis $\lambda, \mu$ of $\Lambda$ such that $\varphi$ is finite on the inner products $\langle\lambda, \lambda\rangle$ and $\langle\lambda, \mu\rangle$.

This lemma will be proved in the next section, and now we shall use it to prove Proposition 4.2.

Recall that an element $\lambda \in \Lambda$ is called primitive if it does not have the form $q \mu$ for an integer $q>1$ and $\mu \in \Lambda$. An element $m \alpha+k \beta$ is primitive if and only if $m$ and $k$ are coprime.

Let us construct two $\mathbb{Q}$-subalgebras $R_{1}$ and $R_{2}$ of $\mathbb{L}$. The algebra $R_{1}$ is obtained by adjoining to $R$ the inverted inner squares of all primitive elements of $\Lambda$ :

$$
R_{1}=R\left[\frac{1}{\langle\lambda, \lambda\rangle} \mid \lambda \in \Lambda \text { is primitive }\right] .
$$

The algebra $R_{2}$ is obtained by adjoining to $R$ the inverted inner products of all pairs of non-proportional primitive elements of $\Lambda$ :

$$
R_{2}=R\left[\frac{1}{\langle\lambda, \mu\rangle} \mid \lambda, \mu \in \Lambda \text { are primitive, } \lambda \neq \pm \mu\right] .
$$

Let us consider the ideal

$$
I_{1}=\left(\frac{1}{\langle\lambda, \lambda\rangle} \mid \lambda \in \Lambda \text { is primitive }\right) \triangleleft R_{1} .
$$

There are two cases: $I_{1}=R_{1}$ and $I_{1} \neq R_{1}$.

If $I_{1} \neq R_{1}$, then there exists a maximal ideal $\mathfrak{m}_{1} \supset I_{1}$. Consider the field $F=$ $R_{1} / \mathfrak{m}_{1}$. Since $R_{1}$ contains $\mathbb{Q}$, we have char $F=0$. By Lemma 3.3, the quotient homomorphism $R_{1} \rightarrow F$ can be extended to a place $\varphi: \mathbb{L} \rightarrow \bar{F} \cup\{\infty\}$. The place $\varphi$ is finite on $R_{1}$ and vanishes on $\mathfrak{m}_{1}$. Hence $\varphi$ is finite on $R$. Besides, by Lemma 3.2, $\varphi$ is infinite on $\langle\lambda, \lambda\rangle$ for all primitive elements $\lambda \in \Lambda$. This contradicts Lemma 4.3. Consequently, this is not the case.

If $I_{1}=R_{1}$, then $1 \in I_{1}$. Hence

$$
1=\sum_{i=1}^{p} \frac{r_{i}}{\left\langle\lambda_{i 1}, \lambda_{i 1}\right\rangle\left\langle\lambda_{i 2}, \lambda_{i 2}\right\rangle \cdots\left\langle\lambda_{i q_{i}}, \lambda_{i q_{i}}\right\rangle},
$$

where $r_{i} \in R, q_{i} \geq 1$, and $\lambda_{i j}$ are primitive elements of $\Lambda$. Multiplying both sides by the product of all denominators, and putting $\lambda_{i j}=m_{i j} \alpha+k_{i j} \beta, m_{i j}, k_{i j} \in \mathbb{Z}$, we 
obtain an algebraic equation on $g_{i j}$ :

$$
\begin{aligned}
& \prod_{i=1}^{p} \prod_{j=1}^{q_{i}}\left(m_{i j}^{2} g_{11}+2 m_{i j} k_{i j} g_{12}+k_{i j}^{2} g_{22}\right) \\
& \quad=r_{1} \prod_{i=2}^{p} \prod_{j=1}^{q_{i}}\left(m_{i j}^{2} g_{11}+2 m_{i j} k_{i j} g_{12}+k_{i j}^{2} g_{22}\right)+\cdots
\end{aligned}
$$

Taking all summands to the left-hand side, we obtain a relation of the form $f\left(g_{11}, g_{12}, g_{22}\right)=0$. Since $q_{i} \geq 1$ for all $i$, we see that

$$
\widehat{f}(X, Y, Z)=\prod_{i=1}^{p} \prod_{j=1}^{q_{i}}\left(m_{i j}^{2} X+2 m_{i j} k_{i j} Y+k_{i j}^{2} Z\right)=\prod_{i=1}^{q}\left(m_{i}^{2} X+2 m_{i} k_{i} Y+k_{i}^{2} Z\right),
$$

where $q=q_{1}+\cdots+q_{p}$. (We have renumbered the pairs $\left(m_{i j}, k_{i j}\right)$ by a single index $i$.)

Similarly, consider the algebra $R_{2}$ and the ideal

$$
I_{2}=\left(\frac{1}{\langle\lambda, \mu\rangle} \mid \lambda, \mu \in \Lambda \text { are primitive, } \lambda \neq \pm \mu\right) .
$$

There are two cases: $I_{2}=R_{2}$ and $I_{2} \neq R_{2}$. Again, $I_{2} \neq R_{2}$ contradicts Lemma 4.3. So we have $1 \in I_{2}$. Then

$$
1=\sum_{i=1}^{l} \frac{s_{i}}{\left\langle\lambda_{i 1}, \mu_{i 1}\right\rangle\left\langle\lambda_{i 2}, \mu_{i 2}\right\rangle \cdots\left\langle\lambda_{i t_{i}}, \mu_{i t_{i}}\right\rangle}
$$

where $s_{i} \in R, t_{i} \geq 1$, and $\lambda_{i j} \neq \pm \mu_{i j}$ are primitive elements of $\Lambda$. Since $\left\langle\lambda_{i j}, \mu_{i j}\right\rangle=$ $A_{i j} g_{11}+B_{i j} g_{12}+C_{i j} g_{22}, A_{i j}, B_{i j}, C_{i j} \in \mathbb{Z}, B_{i j}^{2} \neq 4 A_{i j} C_{i j}$, we obtain an equation $h\left(g_{11}, g_{12}, g_{22}\right)=0$ such that

$$
\widehat{h}(X, Y, Z)=\prod_{j=1}^{t}\left(A_{j} X+B_{j} Y+C_{j} Z\right), \quad B_{j}^{2} \neq 4 A_{j} C_{j} .
$$

Equations (2) and (3) immediately imply that the polynomials $\widehat{f}$ and $\widehat{h}$ have coefficients in $\mathbb{Q}$ and are non-zero. Hence the polynomials $f$ and $h$ are also non-zero. Moreover, $\widehat{f}$ and $\widehat{h}$ both are the products of linear polynomials. It follows from the condition $B_{j}^{2} \neq 4 A_{j} C_{j}$ in (3) that no linear multiple can appear in the decompositions for both $\widehat{f}$ and $\widehat{h}$. Therefore $\widehat{f}$ and $\widehat{h}$ are coprime.

Proof of Theorem 4.1 If the set of numbers $l=\left\{l_{u v}\right\}$ cannot be realized as the set of the squares of the edge lengths of a polyhedral surface $(K, \theta)$, then the set $\mathcal{G}(K, \Lambda, l)$ is empty; hence, the assertion of the theorem is trivial.

Now, suppose that $l$ is the set of the squares of the edge lengths of $(K, \theta)$. Let $\eta: R \rightarrow \mathbb{R}$ be the restriction of the specialization homomorphism $\tau_{\theta}$ to $R$. Since 
$\eta\left(\ell_{u v}\right)=l_{u v}$, the homomorphism $\eta$ is independent of the choice of $\theta$. Let $f, h \in$ $R[X, Y, Z]$ be the polynomials in Proposition 4.2 , and let $\bar{f}, \bar{h} \in \mathbb{R}[X, Y, Z]$ be their images under $\eta$. Since $\widehat{f}, \widehat{h} \in \mathbb{Q}[X, Y, Z]$, and $\left.\eta\right|_{\mathbb{Q}}$ is the identity homomorphism, the leading terms of $\bar{f}$ and $\bar{h}$ are again $\widehat{f}$ and $\widehat{h}$, respectively. But $\widehat{f}$ and $\widehat{h}$ are coprime. Therefore, $\bar{f}$ and $\bar{h}$ are coprime. Hence the set $\mathcal{G}(K, \Lambda, l)$ is contained in the onedimensional variety

$$
\left\{\begin{array}{l}
\bar{f}(X, Y, Z)=0, \\
\bar{h}(X, Y, Z)=0 .
\end{array}\right.
$$

\section{Proof of Lemma 4.3}

Let $V$ be the vertex set of the simplicial complex $K$. A vertex $v \in V$ will be called special if it is connected by an edge of $K$ with another vertex in the orbit $\Lambda v$. If an edge $e$ connects vertices $v$ and $T_{\lambda}(v), \lambda \in \Lambda$, then the edge $T_{\mu}(e)$ connects the vertices $T_{\mu}(v)$ and $T_{\lambda}\left(T_{\mu}(v)\right)$ for every $\mu \in \Lambda$. Hence the property of being special is invariant under the action of $\Lambda$. A $\Lambda$-orbit $\Lambda v \subset V$ will be called special if it consists of special vertices.

Lemma 5.1 Suppose that a vertex $v$ of $K$ is connected by an edge with a vertex $T_{\lambda}(v)$, $\lambda \in \Lambda$. Then $\lambda$ is primitive.

Proof The quotient surface $K / \Lambda$ is homeomorphic to the torus. We have the natural isomorphism $H_{1}(K / \Lambda, \mathbb{Z}) \cong \Lambda$. We shall identify these two groups by this natural isomorphism. Let $e$ be an edge connecting $v$ and $T_{\lambda}(v)$, and let $\gamma$ be the image of $e$ under the quotient mapping $K \rightarrow K / \Lambda$. The endpoints of $e$ lie in the same $\Lambda$-orbit, and no other two points of $e$ lie in the same $\Lambda$-orbit. Hence $\gamma$ is a simple closed curve of homology class $\lambda$. Therefore, $\lambda$ is primitive.

Lemma 5.2 Suppose that $K$ contains at least one special vertex. Then there exists a primitive element $\lambda \in \Lambda$ such that every special vertex $v$ of $K$ is connected by an edge with $T_{\lambda}(v)$.

Proof Let $v$ be a special vertex. Then there exists an element $\lambda \in \Lambda$ such that the vertices $v$ and $T_{\lambda}(v)$ are connected by an edge $e$. By Lemma 5.1, $\lambda$ is primitive.

Let $v^{\prime}$ be any other special vertex. If $v^{\prime}=T_{\mu}(v)$ for a $\mu \in \Lambda$, then $v^{\prime}$ is connected with $T_{\lambda}\left(v^{\prime}\right)$ by an edge $T_{\mu}(e)$. Suppose that $v^{\prime} \notin \Lambda v$. Since $v^{\prime}$ is special, there exists an element $\lambda^{\prime} \in \Lambda$ such that the vertices $v^{\prime}$ and $T_{\lambda^{\prime}}\left(v^{\prime}\right)$ are connected by an edge $e^{\prime}$. Let $\gamma$ and $\gamma^{\prime}$ be the images of $e$ and $e^{\prime}$, respectively, under the quotient mapping $K \rightarrow K / \Lambda$. Then $\gamma$ and $\gamma^{\prime}$ are simple closed curves of the homology classes $\lambda$ and $\lambda^{\prime}$, respectively. Since $\Lambda v^{\prime} \neq \Lambda v$, the simple closed curves $\gamma$ and $\gamma^{\prime}$ are disjoint. Hence either $\lambda^{\prime}=\lambda$ or $\lambda^{\prime}=-\lambda$. Therefore, the vertices $v^{\prime}$ and $T_{\lambda}\left(v^{\prime}\right)$ are joined either by the edge $e^{\prime}$ or by the edge $T_{\lambda}\left(e^{\prime}\right)$.

We shall prove Lemma 4.3 by induction on the number $n$ of non-special $\Lambda$-orbits in $V$. 
Fig. 4 The torus $K / \Lambda$ : (a) decomposition into cylinders, (b) triangulation

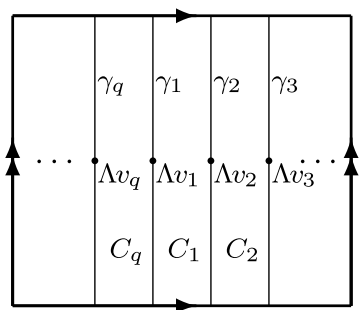

$a$

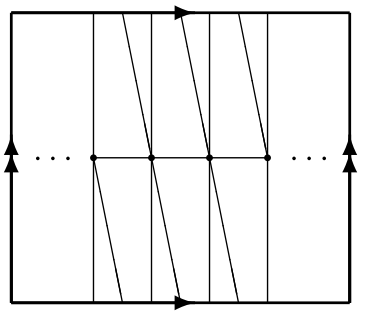

$b$

Fig. 5 The triangulation $K$

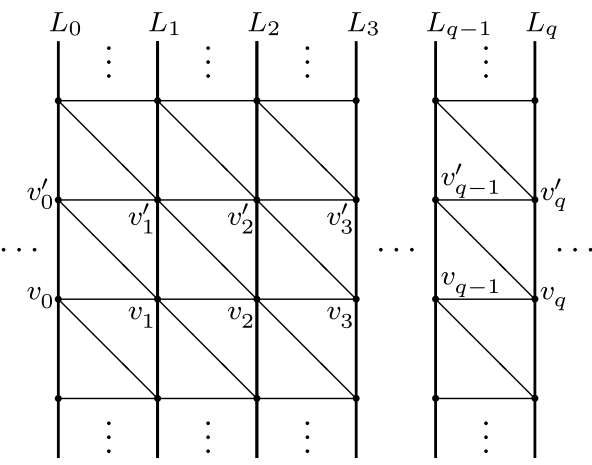

Basis of Induction Suppose that $n=0$. Then all vertices $v \in V$ are special. By Lemma 5.2, there exists a primitive element $\lambda \in \Lambda$ such that $v$ and $T_{\lambda}(v)$ are joined by an edge for every $v \in V$. We denote this edge by $e_{v}$.

The image of $e_{v}$ under the quotient mapping $K \rightarrow K / \Lambda$ is a simple closed curve $\gamma_{\Lambda v}$ of the homology class $\lambda$. (Obviously, this curve depends only on the $\Lambda$ orbit of $v$.) For distinct $\Lambda$-orbits $\Lambda v_{1}$ and $\Lambda v_{2}$, the curves $\gamma_{\Lambda v_{1}}$ and $\gamma_{\Lambda v_{2}}$ are disjoint. Then the curves $\gamma_{\Lambda v}$ decompose the torus $K / \Lambda$ into cylinders as shown in Fig. 4(a). Let $\Lambda v_{1}, \ldots, \Lambda v_{q}$ be all the different $\Lambda$-orbits in $V$, numbered so that the curves $\gamma_{i}=\gamma_{\Lambda v_{i}}$ go successively around the torus $K / \Lambda$. We denote by $C_{i}$ the cylinder bounded by the curves $\gamma_{i}$ and $\gamma_{i+1}$.

The quotient $K / \Lambda$ is an ideal triangulation of the torus, i.e., a triangulation which is not necessarily a simplicial complex. Vertices of $K / \Lambda$ are in one-to-one correspondence with the orbits $\Lambda v, v \in V$. The restriction of the triangulation $K / \Lambda$ to the cylinder $C_{i}$ is a triangulation with exactly two vertices $\Lambda v_{i}$ and $\Lambda v_{i+1}$ whose boundary consists of two edges $\gamma_{i}$ and $\gamma_{i+1}$. Standard calculation of the Euler characteristic yields the result that this triangulation has exactly two faces and four edges. It is easy to see that such an ideal triangulation of the cylinder is unique up to an isomorphism. Hence the triangulation $K / \Lambda$ is isomorphic to the triangulation shown in Fig. 4(b).

Therefore, the triangulation $K$ is isomorphic to the simplicial complex shown in Fig. 5. The pre-image under the quotient mapping $K \rightarrow K / \Lambda$ of every curve $\gamma_{i}$ is a union of countably many lines $L_{i+q j}, j \in \mathbb{Z}$, each homeomorphic to $\mathbb{R}$. The preimage of every cylinder $C_{i}$ is a union of countably many strips $S_{i+q j}, j \in \mathbb{Z}$, each homeomorphic to $\mathbb{R} \times I$. The vertices $v_{i} \in L_{i}$ may be chosen so that the vertices $v_{i}$, 
$v_{i}^{\prime}=T_{\lambda}\left(v_{i}\right)$, and $v_{i+1}$ span a triangle in $S_{i}$ for every $i$. The line $L_{i}$ consists of the vertices $T_{\lambda}^{k}\left(v_{i}\right)$ and the edges $T_{\lambda}^{k}\left(e_{v_{i}}\right), k \in \mathbb{Z}$.

The orbit $\Lambda v_{i}$ is the union of the vertex sets of the lines $L_{i+q j}, j \in \mathbb{Z}$. Let $v_{q}=T_{\mu}\left(v_{0}\right)$. Then $v_{i+q}=T_{\mu}\left(v_{i}\right)$ for every $i$. It easily follows that any vertex in the orbit $\Lambda v_{i}$ has the form $T_{\lambda}^{k} T_{\mu}^{m}\left(v_{i}\right)=T_{k \lambda+m \mu}\left(v_{i}\right)$. Therefore, $\lambda, \mu$ is a basis of $\Lambda$. We have $\langle\lambda, \lambda\rangle=\ell_{v_{0} v_{0}^{\prime}}$ and

$$
\langle\lambda, \mu\rangle=\left\langle\lambda, v_{q}-v_{0}\right\rangle=\sum_{i=0}^{q-1}\left\langle v_{i}^{\prime}-v_{i}, v_{i+1}-v_{i}\right\rangle=\frac{1}{2} \sum_{i=0}^{q-1}\left(\ell_{v_{i} v_{i}^{\prime}}+\ell_{v_{i} v_{i+1}}-\ell_{v_{i}^{\prime} v_{i+1}}\right) .
$$

Since the place $\varphi$ is finite on $\ell_{v w}$ for all edges $[v w]$ of $K$, and char $F \neq 2$, using Lemma 3.2, we conclude that $\varphi$ is finite on $\langle\lambda, \lambda\rangle$ and $\langle\lambda, \mu\rangle$.

Induction Step Let $n$ be the number of non-special $\Lambda$-orbits in $V$. Let $d$ be the smallest degree of a non-special vertex in $V$. (The degree of a vertex is the number of edges incident to it.) We assume that we have already proved the assertion of Lemma 4.3 for all $K$ with strictly smaller $n$, or with the same $n$ and strictly smaller $d$.

We shall consider two cases.

1. Suppose that $K$ contains an 'empty triangle', that is, three vertices $u$, $v$, and $w$ such that $[u v],[v w]$, and $[w u]$ are edges of $K$, but $[u v w]$ is not a triangle of $K$. Since $K$ is homeomorphic to a plane, we see that the curve $\gamma$ consisting of the edges $[u v]$, $[v w]$, and [wu] decomposes $K$ into two parts, one of which is homeomorphic to a disk. We denote the closure of this part by $D$. Then $D$ is a finite subcomplex of $K$.

Lemma 5.3 The interiors of the disks $D_{\lambda}=T_{\lambda}(D), \lambda \in \Lambda$, are disjoint.

Proof Obviously, it is sufficient to prove that the interiors of the disks $D$ and $D_{\lambda}$ are disjoint whenever $\lambda \neq 0$. If $D \supset D_{\lambda}$, then $D \supset D_{k \lambda}$ for all $k>0$. Hence, $D$ contains infinitely many distinct vertices $T_{k \lambda}(u)$, which is impossible, since $D$ is a finite simplicial complex. Therefore, $D \not \supset D_{\lambda}$. Similarly, $D \not \subset D_{\lambda}$. Suppose, $\operatorname{Int}(D) \cap$ $\operatorname{Int}\left(D_{\lambda}\right) \neq \emptyset$; then the intersection $\partial D \cap \partial D_{\lambda}$ must contain at least two distinct points. But this intersection is a subcomplex of $K$. Hence it contains at least two distinct vertices of $K$. This means that two of the three vertices $T_{\lambda}(u), T_{\lambda}(v)$, and $T_{\lambda}(w)$ coincide with two of the three vertices $u, v$, and $w$. Since the action of $\Lambda$ on $K$ is free, we obtain $T_{\lambda}(s) \neq s$ for all vertices $s$, and, if $T_{\lambda}(s)=t$, then $T_{\lambda}(t) \neq s$. Hence, permuting $u, v$, and $w$, we may achieve that $T_{\lambda}(u)=v$ and $T_{\lambda}(v)=w$. Then the vertices $u$ and $w=T_{2 \lambda}(u)$ are connected by an edge in $K$. But this is impossible by Lemma 5.1. Thus, the interiors of $D$ and $D_{\lambda}$ are disjoint.

Since $[u v w]$ is not a triangle of $K$, we see that the interior of $D$ contains at least one vertex $p$. Lemma 5.3 implies that the vertex $p$ is non-special. Consider the simplicial complex $K_{1}$ obtained from $K$ by replacing every subcomplex $T_{\lambda}(D)$ by the triangle $\left[T_{\lambda}(u) T_{\lambda}(v) T_{\lambda}(w)\right]$. It is easy to see that $K_{1}$ is a well-defined simplicial complex that is homeomorphic to a plane and periodic with the same period lattice $\Lambda$. Let $V_{1}$ be the vertex set of $K_{1}$. The number of non-special $\Lambda$-orbits in $V_{1}$ is strictly less 
than the number of non-special $\Lambda$-orbits in $V$, since at least one non-special orbit $\Lambda p$ has been deleted. By the inductive assumption, the assertion of Lemma 4.3 holds for $K_{1}$. Since all edges of $K_{1}$ are edges of $K$, this immediately implies the assertion of Lemma 4.3 for $K$.

2. Suppose that $K$ contains no "empty triangles". We shall use the following lemma, due to Connelly, Sabitov, and Walz [2].

Lemma 5.4 Let $u$ be a vertex of a two-dimensional simplicial manifold $K$, and let $v_{1}, \ldots, v_{d}, d \geq 4$, be all vertices adjacent to $u$, in this cyclic order. Let $\varphi$ be a place that is defined on the field $\mathbb{K}=\mathbb{Q}\left(x_{u}, y_{u}, z_{u}, x_{v_{1}}, y_{v_{1}}, z_{v_{1}}, \ldots, x_{v_{d}}, y_{v_{d}}, z_{v_{d}}\right)$ and is finite on all squares of the edge lengths $\ell_{u v_{i}}, i=1, \ldots, d, \ell_{v_{i} v_{i+1}}, i=1, \ldots, d-1$, and $\ell_{v_{d} v_{1}}$. Then $\varphi$ is finite on at least one of the squares of the diagonal lengths $\ell_{v_{i} v_{i+2}}, i=1, \ldots, d-2$.

We shall take for $u$ a non-special vertex of $K$ of the smallest degree $d$, and we define $v_{1}, \ldots, v_{d}$ as in the above lemma. Since $K$ does not contain 'empty triangles', we see that $d \geq 4$, and $\left[v_{i} v_{i+2}\right]$ are not edges of $K, i=1, \ldots, d-2$. We cannot apply Lemma 5.4 immediately to our place $\varphi: \mathbb{L} \rightarrow F \cup\{\infty\}$, since the field $\mathbb{K}$ is not necessarily a subfield of $\mathbb{L}$. Indeed, some of the vertices $v_{j}$ may belong to the same $\Lambda$-orbits, hence, their coordinates may be mutually dependent in $\mathbb{L}$. Consider the homomorphism

$$
\psi: \mathbb{Q}\left[x_{u}, y_{u}, z_{u}, x_{v_{1}}, y_{v_{1}}, z_{v_{1}}, \ldots, x_{v_{d}}, y_{v_{d}}, z_{v_{d}}\right] \rightarrow \mathbb{L}
$$

taking independent variables $x_{u}, y_{u}, \ldots, z_{v_{d}}$ to the elements of $\mathbb{L}$ denoted by the same letters. By Lemma 3.3, this homomorphism can be extended to a place $\mathbb{K} \rightarrow \overline{\mathbb{L}} \cup$ $\{\infty\}$, which we also denote by $\psi$. Besides, applying Lemma 3.3 to the subring of $\mathbb{L}$ consisting of all elements with finite $\varphi$-values, we see that $\varphi$ can be extended to a place $\bar{\varphi}: \overline{\mathbb{L}} \rightarrow \bar{F} \cup\{\infty\}$. Applying Lemma 5.4 to the composite place

$$
\tilde{\varphi}: \mathbb{K} \stackrel{\psi}{\rightarrow} \overline{\mathbb{L}} \cup\{\infty\} \stackrel{\bar{\varphi}}{\rightarrow} \bar{F} \cup\{\infty\},
$$

we find that there is an $i$ such that $\tilde{\varphi}\left(\ell_{v_{i} v_{i+2}}\right) \neq \infty$. Then $\varphi\left(\ell_{v_{i} v_{i+2}}\right) \neq \infty$.

We replace the two triangles $\left[u v_{i} v_{i+1}\right]$ and $\left[u v_{i+1} v_{i+2}\right]$ by the two triangles $\left[u v_{i} v_{i+2}\right]$ and $\left[v_{i} v_{i+1} v_{i+2}\right]$. Since $\left[v_{i} v_{i+2}\right]$ is not an edge of $K$, we obtain a simplicial complex homeomorphic to a plane. To keep the simplicial complex $\Lambda$-periodic we simultaneously replace every pair of triangles $T_{\lambda}\left(\left[u v_{i} v_{i+1}\right]\right)$ and $T_{\lambda}\left(\left[u v_{i+1} v_{i+2}\right]\right)$ by the pair $T_{\lambda}\left(\left[u v_{i} v_{i+2}\right]\right)$ and $T_{\lambda}\left(\left[v_{i} v_{i+1} v_{i+2}\right]\right)$. (All triangles $T_{\lambda}\left(\left[u v_{i} v_{i+1}\right]\right)$ and $T_{\lambda}\left(\left[u v_{i+1} v_{i+2}\right]\right), \lambda \in \Lambda$, are pairwise distinct. Hence we actually can perform all of these flips simultaneously.) We denote the resulting simplicial complex by $K^{\prime}$. All edges of $K^{\prime}$ are edges of $K$ except for the edges $T_{\lambda}\left(\left[v_{i} v_{i+2}\right]\right)$. Since $\varphi\left(\ell_{v_{i} v_{i+2}}\right) \neq \infty$, we see that $\varphi$ is finite on all squares of the edge lengths of $K^{\prime}$.

The vertex set of $K^{\prime}$ coincides with the vertex set $V$ of $K$. An edge of $K$ is not an edge of $K^{\prime}$ if and only if it coincides with one of the edges $T_{\lambda}\left(\left[u v_{i+1}\right]\right)$. Since $u$ is non-special, any such edge connects vertices belonging to distinct $\Lambda$-orbits. Hence any vertex $w$ that is special in $K$ is also special in $K^{\prime}$. Since $u$ is non-special in $K$, we see that none of the vertices $T_{\lambda}\left(v_{j}\right), \lambda \in \Lambda, j=1, \ldots, d$, coincides with $u$. Hence the 
degree of $u$ in $K^{\prime}$ is equal to $d-1$ and $u$ is non-special in $K^{\prime}$. Therefore, the number of non-special $\Lambda$-orbits of vertices of $K^{\prime}$ is not greater than $n$, and the smallest degree of a non-special vertex of $K^{\prime}$ is strictly less than $d$. Applying the inductive assumption for $K^{\prime}$, we obtain the result that there exists a basis $\lambda, \mu$ of $\Lambda$ such that $\varphi$ is finite on $\langle\lambda, \lambda\rangle$ and $\langle\lambda, \mu\rangle$, which completes the proof of Lemma 4.3.

Acknowledgements The first author was partially supported by the Russian Foundation for Basic Research (projects 12-01-31444 and 13-01-12469), by a grant of the President of the Russian Federation (project MD-4458.2012.1), by a grant of the Government of the Russian Federation (project 11.G34.31.0053), by a program of the Branch of Mathematical Sciences of the Russian Academy of Sciences, and by a grant from Dmitry Zimin's "Dynasty" foundation.

The second author was partially supported by the Russian Foundation for Basic Research (projects 12-01-31342 and 12-01-00704) and by the Ministry of Education and Science of the Russian Federation (project 8214).

The authors are grateful to V.M. Buchstaber and I.Kh. Sabitov for useful discussions.

\section{References}

1. Borcea, C.S., Streinu, I.: Periodic frameworks and flexibility. Proc. R. Soc. A 466, 2633-2649 (2010)

2. Connelly, R., Sabitov, I., Walz, A.: The bellows conjecture. Beitr. Algebra Geom. 38(1), 1-10 (1997)

3. Gaifullin, A.A.: Sabitov polynomials for volumes of polyhedra in four dimensions. Adv. Math. 252, 586-611 (2014). arXiv:1108.6014

4. Gaifullin, A.A.: Generalization of Sabitov's theorem to polyhedra of arbitrary dimensions (2012). arXiv: 1210.5408

5. Lang, S.: Introduction to Algebraic Geometry. Addison-Wesley, Reading (1972)

6. Malestein, J., Theran, L.: Generic combinatorial rigidity of periodic frameworks. Adv. Math. 233(1), 291-331 (2013). arXiv:1008.1837

7. Pak, I.: Lectures on discrete and polyhedral geometry. Monograph draft (2010). Available at http://www.math.ucla.edu/ pak/book.htm

8. Ross, E., Schulze, B., Whiteley, W.: Finite motions from periodic frameworks with added symmetry. Int. J. Solids Struct. 48(11-12), 1711-1729 (2011)

9. Sabitov, I.Kh.: Volume of a polyhedron as a function of its metric. Fundam. Appl. Math. 2(4), 12351246 (1996) (in Russian)

10. Sabitov, I.Kh.: The volume as a metric invariant of polyhedra. Discrete Comput. Geom. 20(4), 405425 (1998)

11. Stachel, H.: Remarks on flexible quad meshes. In: Proc. BALTGRAF-11, Eleventh. Internat. Conf., Tallin, Estonia (2011)

12. Stachel, H.: A flexible planar tessellation with a flexion tiling a cylinder of revolution. J. Geom. Graph. 16(2), 153-170 (2012) 